\title{
ON THE ASYMPTOTICAL REGULARIZATION WITH CONVEX CONSTRAINTS FOR INVERSE PROBLEMS
}

\author{
MIN ZHONG AND WEI WANG
}

\begin{abstract}
In this paper, we consider the asymptotical regularization with convex constraints for nonlinear ill-posed problems. The method allows to use non-smooth penalty terms, including the L1-like and the total variation-like penalty functionals, which are significant in reconstructing special features of solutions such as sparsity and piecewise constancy. Under certain conditions we give convergence properties of the methods. Moreover, we propose RungeKutta type methods to discrete the initial value problems to construct new type iterative regularization methods.
\end{abstract}

\section{INTRODUCTION}

Consider the ill-posed operator equation

$$
F(x)=y,
$$

where $F: \mathcal{D}(F) \subset \mathcal{X} \rightarrow \mathcal{Y}$ is a nonlinear operator, $\mathcal{X}, \mathcal{Y}$ are infinite dimensional Hilbert spaces. Instead of exact data $y$, in practice we are given only noisy data $y^{\delta}$ satisfying

$$
\left\|y-y^{\delta}\right\| \leqslant \delta
$$

with known noise level $\delta$.

In order to find the solution of (1.1) with desired feature such as sparsity and piecewise constancy, one may introduce non-smooth penalty functions, such as the $L^{1}$ and total variation like functions 4, 5. In recent years, the relative iterative regularization has received tremendous attention, while Landweber type iteration is one of the most fundamental methods 2, 16.

For a proper, lower semi-continuous, uniformly convex function as penalty term $\Theta: \mathcal{X} \rightarrow(-\infty,+\infty)$ given the initial pairs $\left(\xi_{0}^{\delta}, x_{0}^{\delta}\right)=\left(\xi_{0}, \nabla \Theta^{*}\left(\xi_{0}\right)\right)$, the Landweber iteration with penalty $\Theta$ takes the form

$$
\begin{aligned}
& \xi_{n+1}^{\delta}=\xi_{n}^{\delta}-\mu_{n}^{\delta} F^{\prime}\left(x_{n}^{\delta}\right)^{*}\left(F\left(x_{n}^{\delta}\right)-y^{\delta}\right), \\
& x_{n+1}^{\delta}=\nabla \Theta^{*}\left(\xi_{n+1}^{\delta}\right), \quad n=0,1,2, \ldots
\end{aligned}
$$

where $F^{\prime}(\cdot)^{*}$ denotes the adjoint of $F^{\prime}, \mu_{n}^{\delta}$ is the step-size and $\Theta^{*}: \mathcal{X} \rightarrow(-\infty, \infty]$ is the Legendre-Fenchel conjugate of $\Theta$. The advantage of this method is the freedom on the choice of $\Theta$ so that the method can be utilized in detecting different features of the sought solution. Extensive researches have been carried out to study above Landweber-type iteration, its accelerated version and its modification. The convergence analysis of the iterative methods has been given under discrepancy principle or Heuristic choice rule [15, 19, 10, 29, 23, 28, 27.

In this paper, we investigate the methods of asymptotical regularization, which can be considered as the continuous analogue of iteration (1.3). By introducing an

Date: October 12, 2021. 
artificial time variable, a regularized approximation pair $\left(\xi^{\delta}(T), x^{\delta}(T)\right)$ is obtained by solving the initial value problem

$$
\begin{aligned}
\frac{d \xi^{\delta}(t)}{d t} & =-F^{\prime}\left(x^{\delta}(t)\right)^{*}\left(F\left(x^{\delta}(t)\right)-y^{\delta}\right), \quad 0<t \leqslant T, \\
x^{\delta}(t) & =\nabla \Theta^{*}\left(\xi^{\delta}(t)\right),
\end{aligned}
$$

with initial value $\left(\xi^{\delta}(0), x^{\delta}(0)\right)=\left(\xi_{0}, \nabla \Theta^{*}\left(\xi_{0}\right)\right)$. Note that, when take $\Theta(x)=$ $\frac{1}{2}\|x\|^{2}$, then (1.4) became the asymptotical regularization in 24]

$$
\frac{d x^{\delta}(t)}{d t}=-F^{\prime}\left(x^{\delta}(t)\right)^{*}\left(F\left(x^{\delta}(t)\right)-y^{\delta}\right), \quad 0<t \leqslant T .
$$

with initial value $x^{\delta}(0)=x_{0}$. In [24, Ulrich Tautenhahn give convergence properties of method (1.5) and derive stability estimates. It also has been show that $T$ plays the role of the regularization parameter and can be chosen by a generalized discrepancy principle. For the autonomous ODE (1.5), Runge-Kutta method can be used and the new type iterative regularization methods can be constructed. The theory of the RK regularization method has been well-developed for linear inverse problems 22, 26, and for nonlinear inverse problems [17, 3], as some special cases, such as explicit Euler method(Landweber iteration) 13, explicit 2-stage R-K Landweber method 17, implicit Euler method (Levenberg-Marquardt iteration) [12.

Recently, there has been a great interest in analysing such dynamical flows. In [26], variational source conditions have been established for general spectral regularization methods which yield convergence rates. In [18, the authors interpreted steady linear statistical inverse problems with white noise, and introduced a stochastic differential equation system. In 1, the authors applied the general theory of convergence rates to the three, well studied examples, including Showalter's method, heavy ball method and the vanishing viscosity method. Besides general spectral analysis and source condition, the conditional stability assumption was also a efficient tool to analyze the convergence rate, which was fist proposed in 7 , where the convergence rate of the Tikhonov regularization in Hilbert spaces was proved, then, the results have been extended to Banach spaces in [6. In [11, the authors analyzed a nonlinear Landweber iteration and proved its local convergence rates under the Hölder continuity of the inverse mapping. The latest related work was 27, the authors discussed the convergence rate of Landweber-type iteration in Banach spaces.

In this manuscript, we will analyze the regularization property of asymptotical regularization (1.4) through a proper choice of the terminating time. The paper is organized as follows. In section 2, we give some preliminaries from convex analysis. In section 3, we present the detailed analysis of convergence and regularization properties of the asymptotical regularization (1.4) including the convergence rate under conditional stability assumption.

\section{TOOLS FROM CONVEX ANALYSIS}

Given a convex function $\Theta: \mathcal{X} \rightarrow(-\infty, \infty]$, we use $\partial \Theta(x)$ to denote the subdifferential of $\Theta$ at $x \in \mathcal{X}$, i.e.

$$
\partial \Theta(x):=\{\xi \in \mathcal{X}: \Theta(\bar{x})-\Theta(x)-\langle\xi, \bar{x}-x\rangle \geqslant 0 \text { for all } \bar{x} \in \mathcal{X}\} .
$$

Let $\mathcal{D}(\Theta):=\{x \in \mathcal{X}: \Theta(x)<\infty\}$ be its effective domain and let

$$
\mathcal{D}(\partial \Theta):=\{x \in \mathcal{D}(\Theta): \partial \Theta(x) \neq \varnothing\} .
$$

The Bregman distance induced by $\Theta$ at $x$ in the direction $\xi \in \partial \Theta(x)$ is defined by

$$
D_{\xi} \Theta(\bar{x}, x):=\Theta(\bar{x})-\Theta(x)-\langle\xi, \bar{x}-x\rangle, \quad \forall \bar{x} \in \mathcal{X}
$$


which is always nonnegative and satisfies the identity

$$
D_{\xi_{2}} \Theta\left(x, x_{2}\right)-D_{\xi_{1}} \Theta\left(x, x_{1}\right)=D_{\xi_{2}} \Theta\left(x_{1}, x_{2}\right)+\left\langle\xi_{2}-\xi_{1}, x_{1}-x\right\rangle
$$

for all $x \in \mathcal{D}(\Theta), x_{1}, x_{2} \in \mathcal{D}(\partial \Theta)$, and $\xi_{1} \in \partial \Theta\left(x_{1}\right), \xi_{2} \in \partial \Theta\left(x_{2}\right)$.

A proper convex function $\Theta: \mathcal{X} \rightarrow(-\infty, \infty]$ is called uniformly convex if there exists a strictly increasing function $h:[0, \infty) \rightarrow[0, \infty)$ with $h(0)=0$ such that

$$
\Theta(\lambda \bar{x}+(1-\lambda) x)+\lambda(1-\lambda) h(\|x-\bar{x}\|) \leqslant \lambda \Theta(\bar{x})+(1-\lambda) \Theta(x)
$$

for all $\bar{x}, x \in \mathcal{X}$ and $\lambda \in[0,1]$. If $h(t)=c_{0} t^{p}$ for some $c_{0}>0$ and $p>1$ in (2.2), then $\Theta$ is called $p$-convex.

For a proper lower semi-continuous convex function $\Theta: \mathcal{X} \rightarrow(-\infty, \infty]$, its Legendre-Fenchel conjugate is defined by

$$
\Theta^{*}(\xi):=\sup _{x \in \mathcal{X}}\{\langle\xi, x\rangle-\Theta(x)\}, \quad \xi \in \mathcal{X}
$$

which is also proper, lower semi-continuous, and convex. If $\mathcal{X}$ is reflexive, then

$$
\xi \in \partial \Theta(x) \Longleftrightarrow x \in \partial \Theta^{*}(\xi) \Longleftrightarrow \Theta(x)+\Theta^{*}(\xi)=\langle\xi, x\rangle .
$$

For detailed introduction of convex analysis, we refer to 25], here we list some properties.

Proposition 2.1. For a proper lower semi-continuous convex function $\Theta$ and $p>$ 1 , the following statements hold true:

(i) The $\Theta$ is p-convex if and only if

$$
D_{\xi} \Theta(\bar{x}, x) \geqslant c_{0}\|x-\bar{x}\|^{p}, \quad \forall \bar{x} \in \mathcal{D}(\Theta), x \in \mathcal{D}(\partial \Theta), \quad \xi \in \partial \Theta(x) .
$$

(ii) If $\Theta$ is $p$-convex with $p \geqslant 2$, then $\mathcal{D}\left(\Theta^{*}\right)=\mathcal{X}, \Theta^{*}$ is Fréchet differentiable and its gradient $\nabla \Theta^{*}: \mathcal{X} \rightarrow \mathcal{X}$ satisfies

$$
\left\|\nabla \Theta^{*}\left(\xi_{1}\right)-\nabla \Theta^{*}\left(\xi_{2}\right)\right\| \leqslant\left(\frac{\left\|\xi_{1}-\xi_{2}\right\|}{2 c_{0}}\right)^{\frac{1}{p-1}}, \quad \forall \xi_{1}, \xi_{2} \in \mathcal{X} .
$$

(iii) If $\mathcal{X}$ is reflexive and $\Theta$ is $p$-convex with $p \geqslant 2$, then

$$
x=\nabla \Theta^{*}(\xi) \Longleftrightarrow \xi \in \partial \Theta(x) \Longleftrightarrow x=\arg \min _{z \in \mathcal{X}}\{\Theta(z)-\langle\xi, z\rangle\} .
$$

(iv) If $\Theta$ is p-convex, then for any pairs $(x, \xi)$ and $(\bar{x}, \bar{\xi})$ with $x, \bar{x} \in \mathcal{D}(\partial \Theta), \xi \in$ $\partial \Theta(x), \bar{\xi} \in \partial \Theta(\bar{x})$, then

$$
D_{\xi} \Theta(\bar{x}, x) \leqslant \frac{1}{p^{*}\left(2 c_{0}\right)^{p *-1}}\|\xi-\bar{\xi}\|^{p *} .
$$

where $p^{*}=p /(p-1)$ be the conjugate exponent of $p$.

Assumption 2.2. During this manuscript, we provide the following assumptions

(a) Let $\Theta: \mathcal{X} \rightarrow(-\infty, \infty]$ be a proper, weak lower semi-continuous, and $p$ convex functional with $p>1$ such that the condition (2.4) is satisfied for some $c_{0}>0$.

(b) There exists $\rho>0, x_{0} \in \mathcal{X}$ and $\xi_{0} \in \partial \Theta\left(x_{0}\right)$ such that $B_{2 \rho}\left(x_{0}\right) \subset \mathcal{D}(F)$, where $B_{2 \rho}\left(x_{0}\right)$ denotes the closed ball around $x_{0}$ with radius $2 \rho$. In addition, assume (1.1) has a solution $x^{*} \in \mathcal{D}(\Theta)$ with

$$
D_{\xi_{0}} \Theta\left(x^{*}, x_{0}\right) \leqslant c_{0} \rho^{p} .
$$

(c) The operator $F$ is continuous and weakly closed on $\mathcal{D}(F)$. 
(d) There exists a family of bounded linear operators $\{L(x): \mathcal{X} \rightarrow \mathcal{Y}\}_{x \in B_{2 \rho}\left(x_{0}\right) \cap \mathcal{D}(\Theta)}$ such that $x \rightarrow L(x)$ is continuous on $B_{2 \rho}\left(x_{0}\right) \cap \mathcal{D}(\Theta)$ and there is $0 \leqslant \eta<1$ such that tangential cone condition

$$
\|F(x)-F(\bar{x})-L(\bar{x})(x-\bar{x})\|<\eta\|F(x)-F(\bar{x})\|
$$

holds true for all $x, \bar{x} \in B_{2 \rho}\left(x_{0}\right) \cap \mathcal{D}(\Theta)$. Moreover, there is a constant $C_{0}>0$ such that

$$
\|L(x)\|_{\mathcal{X} \rightarrow \mathcal{Y}} \leqslant C_{0}, \quad \forall x \in B_{2 \rho}\left(x_{0}\right) .
$$

\section{Asymptotical REgularization With CONVEX PENALTY}

In this paper, we investigate the method of asymptotical regularization, which is the continuous analogue of (1.3). In this section a regularized approximation $x^{\delta}(T)$ of $x^{*}$ is obtained by solving the coupling initial value problem

$$
\begin{aligned}
\frac{d \xi^{\delta}(t)}{d t} & =-L\left(x^{\delta}(t)\right)^{*}\left(F\left(x^{\delta}(t)\right)-y^{\delta}\right), \quad 0<t \leqslant T, \\
x(t) & =\nabla \Theta^{*}\left(\xi^{\delta}(t)\right), \\
x^{\delta}(0) & =x_{0}, \quad \xi^{\delta}(0)=\partial \Theta\left(x_{0}\right) .
\end{aligned}
$$

Here and subsequently, the $L(x)$ is a family of bounded linear operators from $\mathcal{X}$ to $\mathcal{Y}$ satisfying Assumption 2.2 (d) and functional $\Theta$ satisfying Assumption 2.2 (a). The $T$ plays the role of the regularization parameter.

3.1. Convergence results. In this subsection, we study some convergence properties of (3.1), the basic principle for proof have been developed in [24], where corresponding results have been proved for classical Landweber asymptotical regularization in Hilbert space.

Lemma 3.1. Let Assumption 2.2 hold true. Let $\left(\xi^{\delta}(T), x^{\delta}(T)\right)$ be the solution of (3.1) for $T>0$. Then the approximated solution $x^{\delta}(T)$ and the residual $\| F\left(x^{\delta}(T)\right)$ $y^{\delta} \|$ are continuous with respect to $T$.

Proof. Referring to Proposition 2.1 (ii),

$$
\begin{aligned}
\lim _{\Delta T \rightarrow 0}\left\|x^{\delta}(T+\Delta T)-x^{\delta}(T)\right\| & =\lim _{\Delta T \rightarrow 0}\left\|\nabla \Theta^{*}\left(\xi^{\delta}(T+\Delta T)\right)-\nabla \Theta^{*}\left(\xi^{\delta}(T)\right)\right\| \\
& \leqslant \lim _{\Delta T \rightarrow 0}\left(\frac{\left\|\xi^{\delta}(T+\Delta T)-\xi^{\delta}(T)\right\|}{2 c_{0}}\right)^{\frac{1}{p-1}} \\
& =\lim _{\Delta T \rightarrow 0}\left(\left\|\frac{\xi^{\delta}(T+\Delta T)-\xi^{\delta}(T)}{\Delta T}\right\| \cdot \frac{\Delta T}{2 c_{0}}\right)^{\frac{1}{p-1}}
\end{aligned}
$$

Since

$$
\lim _{\Delta T \rightarrow 0}\left\|\frac{\xi^{\delta}(T+\Delta T)-\xi^{\delta}(T)}{\Delta T}\right\|=\left.\frac{d \xi^{\delta}(t)}{d t}\right|_{t=T}
$$

exists and $\lim _{\Delta T \rightarrow 0} \frac{\Delta T}{2 c_{0}}=0$. This implies $x^{\delta}(T)$ is continuous. With the continuity of $F$ the residual is continuous as well.

Proposition 3.2. Let Assumption 2.2 hold true and $\left(\xi^{\delta}(T), x^{\delta}(T)\right)$ be the solution of (3.1) for $T>0$. Let $\hat{x}$ be any solution of (1.1) satisfying $D_{\xi_{0}} \Theta\left(\hat{x}, x_{0}\right) \leqslant c_{0} \rho^{p}$. Define

$$
\varphi(T):=D_{\xi^{\delta}(T)} \Theta\left(\hat{x}, x^{\delta}(T)\right) .
$$

If $x^{\delta}(T) \in B_{2 \rho}\left(x_{0}\right)$, then $\varphi(T)$ is differentiable and

$$
\varphi^{\prime}(T) \leqslant-(1-\eta)\left\|F\left(x^{\delta}(T)\right)-y\right\|^{2}+\delta(1+\eta)\left\|F\left(x^{\delta}(T)\right)-y^{\delta}\right\| .
$$


In the case $\delta=0$,

$$
\int_{0}^{\infty}\|F(x(\tau))-y\|^{2} d \tau<\frac{1}{1-\eta} D_{\xi_{0}} \Theta\left(\hat{x}, x_{0}\right),
$$

and

$$
\lim _{T \rightarrow \infty}\|F(x(T))-y\|=0 .
$$

Proof. Referring to (2.1), we have

$$
\begin{aligned}
& \lim _{\Delta T \rightarrow 0} \frac{\varphi(T+\Delta T)-\varphi(T)}{\Delta T} \\
= & \lim _{\Delta T \rightarrow 0} \frac{D_{\xi^{\delta}(T+\Delta T)} \Theta\left(\hat{x}, x^{\delta}(T+\Delta T)\right)-D_{\xi^{\delta}(T)} \Theta\left(\hat{x}, x^{\delta}(T)\right)}{\Delta T} \\
= & \lim _{\Delta T \rightarrow 0} \frac{D_{\xi^{\delta}(T+\Delta T)} \Theta\left(x^{\delta}(T), x^{\delta}(T+\Delta T)\right)+\left\langle\xi^{\delta}(T+\Delta T)-\xi^{\delta}(T), x^{\delta}(T)-\hat{x}\right\rangle}{\Delta T} .
\end{aligned}
$$

The application of Proposition 2.1(iv) provides the estimate of the first term

$$
\begin{aligned}
& \lim _{\Delta T \rightarrow 0} \frac{D_{\xi^{\delta}(T+\Delta T)} \Theta\left(x^{\delta}(T), x^{\delta}(T+\Delta T)\right)}{\Delta T} \\
= & \lim _{\Delta T \rightarrow 0} \frac{(\Delta T)^{p^{*}-1}}{p^{*}\left(2 c_{0}\right)^{p^{*}-1}}\left\|\frac{\xi^{\delta}(T+\Delta T)-\xi^{\delta}(T)}{\Delta T}\right\|^{p^{*}} .
\end{aligned}
$$

Referring to (3.2) again, the above estimate converges to 0. For the second term

$$
\begin{aligned}
& \lim _{\Delta T \rightarrow 0}\left\langle\frac{\xi^{\delta}(T+\Delta T)-\xi^{\delta}(T)}{\Delta T}, x^{\delta}(T)-\hat{x}\right\rangle \\
= & \left\langle\frac{d \xi^{\delta}(T)}{d T}, x^{\delta}(T)-\hat{x}\right\rangle \\
= & -\left\langle L\left(x^{\delta}(T)\right)^{*}\left(F\left(x^{\delta}(T)\right)-y^{\delta}\right), x^{\delta}(T)-\hat{x}\right\rangle \\
= & -\left\langle\left(F\left(x^{\delta}(T)\right)-y^{\delta}\right), L\left(x^{\delta}(T)\right)\left(x^{\delta}(T)-\hat{x}\right)\right\rangle \\
= & -\left\|F\left(x^{\delta}(T)\right)-y^{\delta}\right\|^{2}+\left\langle F\left(x^{\delta}(T)\right)-y^{\delta}, F\left(x^{\delta}(T)\right)-y^{\delta}-L\left(x^{\delta}(T)\right)\left(x^{\delta}(T)-\hat{x}\right)\right\rangle \\
= & -\left\|F\left(x^{\delta}(T)\right)-y^{\delta}\right\|^{2}+\delta\left\|F\left(x^{\delta}(T)\right)-y^{\delta}\right\|+\left\langle F\left(x^{\delta}(T)\right)-y^{\delta}, y-F\left(x^{\delta}(T)\right)-L\left(x^{\delta}(T)\right)\left(\hat{x}-x^{\delta}(T)\right)\right\rangle
\end{aligned}
$$

Since, assume $x^{\delta}(T) \in B_{2 \rho}\left(x_{0}\right)$, we have

$$
\left\|y-F\left(x^{\delta}(T)\right)-L\left(x^{\delta}(T)\right)\left(\hat{x}-x^{\delta}(T)\right)\right\| \leqslant \eta\left\|y-F\left(x^{\delta}(T)\right)\right\| .
$$

Putting it into above estimates, it is consequently that $\varphi(t)$ is differentiable and

$$
\begin{aligned}
\varphi^{\prime}(T) & =\lim _{\Delta T \rightarrow 0} \frac{\varphi(T+\Delta T)-\varphi(T)}{\Delta T} \\
& \leqslant-\left\|F\left(x^{\delta}(T)\right)-y^{\delta}\right\|^{2}+\delta\left\|F\left(x^{\delta}(T)\right)-y^{\delta}\right\|+\eta\left\|F\left(x^{\delta}(T)\right)-y^{\delta}\right\|\left(\delta+\left\|F\left(x^{\delta}(T)\right)-y^{\delta}\right\|\right) \\
& =-(1-\eta)\left\|F\left(x^{\delta}(T)\right)-y^{\delta}\right\|^{2}+\delta(1+\eta)\left\|F\left(x^{\delta}(T)\right)-y^{\delta}\right\| .
\end{aligned}
$$

In the case $\delta=0$, by integration from 0 to $\infty$, we have

$$
(1-\eta) \int_{0}^{\infty}\left\|F\left(x^{\delta}(\tau)\right)-y\right\|^{2} d \tau \leqslant-\int_{0}^{\infty} \varphi^{\prime}(\tau) d \tau
$$

which gives (3.5) and consequently (3.6).

Proposition 3.2 shows that, the Bregman distance $D_{\xi^{\delta}(T)} \Theta\left(\hat{x}, x^{\delta}(T)\right)$ as a function of $T$ is monotonically decreasing, as far as $\left\|F\left(x^{\delta}(T)\right)-y^{\delta}\right\|>\tau \delta$ is holding 
with $\tau=(1+\eta) /(1-\eta)$. Hence, it makes sense to choose the regularization parameter by following discrepancy principle, i.e., $T=T^{*}$ is a solution of the nonlinear equation

$$
h(T)=\left\|F\left(x^{\delta}(T)\right)-y^{\delta}\right\|-\tau \delta=0 \text { with } \tau>(1+\eta) /(1-\eta) .
$$

In the following proposition, we prove equation (3.8) has a solution.

Proposition 3.3. Under the condition of Proposition 3.2, if $\left\|F\left(x_{0}\right)-y^{\delta}\right\|>\tau \delta$, then the nonlinear equation $h(T)=0$ exists at least one solution $T=T^{*}$.

Proof. Referring to Lemma 3.1 the function $h(T)$ is continuous, and $h(0)=$ $\left\|F\left(x_{0}\right)-y^{\delta}\right\|-\tau \delta>0$. It is sufficient to show there exists a $\bar{T}$, such that $h(\bar{T}) \leqslant 0$. In contrast, we assume $h(T)>0$ for all $T<\infty$, i.e.,

$$
\left\|F\left(x^{\delta}(T)\right)-y^{\delta}\right\|>\tau \delta, \quad \text { for all } T \geqslant 0 .
$$

Combining this with Proposition 3.2 for all $T \geqslant 0$,

$$
\begin{aligned}
\varphi^{\prime}(T) & \leqslant-(1-\eta)\left\|F\left(x^{\delta}(T)\right)-y\right\|^{2}+\delta(1+\eta)\left\|F\left(x^{\delta}(T)\right)-y^{\delta}\right\| \\
& <-\left(1-\eta-\frac{1+\eta}{\tau}\right)\left\|F\left(x^{\delta}(t)\right)-y^{\delta}\right\|^{2} .
\end{aligned}
$$

By integration, it follows that,

$$
\varphi(T)-\varphi(0) \leqslant-\left(1-\eta-\frac{1+\eta}{\tau}\right) \int_{0}^{T}\left\|F\left(x^{\delta}(\tau)\right)-y^{\delta}\right\|^{2} d \tau,
$$

this implies

$$
\left(1-\eta-\frac{1+\eta}{\tau}\right) \int_{0}^{T}\left\|F\left(x^{\delta}(t)\right)-y^{\delta}\right\|^{2} d t \leqslant \varphi(0)
$$

Thus,

$$
\left(1-\eta-\frac{1+\eta}{\tau}\right) T \tau^{2} \delta^{2}<D_{\xi_{0}} \Theta\left(\hat{x}, x_{0}\right) \leqslant c_{0} \rho^{p} .
$$

This contradicts with the assumption when $T$ is large.

Remark 3.4. Since $\varphi^{\prime}(T) \leqslant 0$ for $T \leqslant T^{*}$, we obtain that

$$
D_{\xi^{\delta}(T)} \Theta\left(\hat{x}, x^{\delta}(T)\right) \leqslant D_{\xi_{0}} \Theta\left(\hat{x}, x_{0}\right) \leqslant c_{0} \rho^{p} .
$$

Applying p-convexity of $\Theta$, it is consequently

$$
\left\|\hat{x}-x^{\delta}(T)\right\| \leqslant \rho .
$$

Then, referring to triangle inequality, we find the solution $x^{\delta}(T)$ remains in $B_{2 \rho}\left(x_{0}\right) \subset$ $D(F)$. Furthermore, in the case $\delta=0, x(T)$ remains in $B_{2 \rho}\left(x_{0}\right)$ for all $T<\infty$, this is because $\frac{d}{d T} D_{\xi(T)} \Theta(\hat{x}, x(T))<0$ for all $T<\infty$.

In the next, we prove that the solution $x(T)$ of (3.1) converges (for $T \rightarrow \infty$ ) to a solution of (1.1).

Theorem 3.5. Let Assumption 2.2 hold true and let $(\xi(T), x(T))$ be the solution of (3.1) in the noisefree case for $T>0$. Then, there exists a solution $\bar{x} \in B_{2 \rho}\left(x_{0}\right) \cap$ $D(\Theta)$ of (1.1) such that

$$
\lim _{T \rightarrow \infty}\|x(T)-\bar{x}\|=0
$$

Proof. We define function $\theta(T)$ such that

$$
\|F(x(\theta(T)))-y\|=\min _{0 \leqslant t \leqslant T}\|F(x(t))-y\| .
$$

It is obvious that the function $\theta(T)$ is continuous, monotonically nondecreasing and can be bounded by $T$. Moreover $\theta(T) \rightarrow \infty$ as $T \rightarrow \infty$. First, we prove the 
sequence $x(\theta(T))$ converges as $T \rightarrow \infty$. To this end, we will prove that for any $\varepsilon>0$, there exists $T_{0}>0$, such that for arbitrary $T_{2}>T_{1}>T$, there holds

$$
D_{\xi\left(\theta\left(T_{1}\right)\right)} \Theta\left(x\left(\theta\left(T_{2}\right)\right), x\left(\theta\left(T_{1}\right)\right)\right)<\varepsilon .
$$

Let $\hat{x}$ be any solution of (1.1) satisfying $D_{\xi_{0}} \Theta\left(\hat{x}, x_{0}\right) \leqslant c_{0} \rho^{p}$, referring to (2.1), we can rewrite the term $D_{\xi\left(\theta\left(T_{1}\right)\right)} \Theta\left(x\left(\theta\left(T_{2}\right)\right), x\left(\theta\left(T_{1}\right)\right)\right)$ as

$$
D_{\xi\left(\theta\left(T_{2}\right)\right)} \Theta\left(\hat{x}, x\left(\theta\left(T_{2}\right)\right)\right)-D_{\theta\left(T_{1}\right)} \Theta\left(\hat{x}, \theta\left(T_{1}\right)\right)+\left\langle\xi\left(\theta\left(T_{1}\right)\right)-\xi\left(\theta\left(T_{2}\right)\right), x\left(\theta\left(T_{2}\right)\right)-\hat{x}\right\rangle .
$$

Since $\left\{D_{\xi(T)} \Theta(\hat{x}, x(T))\right\}$ is strictly decreasing for $T>0$, there exists a constant $a \geqslant 0$ and $\bar{T}>0$, such that for all $T>\bar{T}$, the first and second terms can be bounded as

$$
a-\frac{\varepsilon}{3}<D_{\xi(\theta(T))} \Theta(\hat{x}, x(\theta(T)))<a+\frac{\varepsilon}{3} .
$$

For the third term of (3.11), the fundamental theorem of calculus provides

$$
\begin{aligned}
\left|\left\langle\xi\left(\theta\left(T_{1}\right)\right)-\xi\left(\theta\left(T_{2}\right)\right), x\left(\theta\left(T_{2}\right)\right)-\hat{x}\right\rangle\right| & \leqslant\left|\left\langle\int_{\theta\left(T_{1}\right)}^{\theta\left(T_{2}\right)} L(x(s))^{*}(y-F(x(s))) d s, x\left(\theta\left(T_{2}\right)\right)-\hat{x}\right\rangle\right| \\
& \leqslant \int_{\theta\left(T_{1}\right)}^{\theta\left(T_{2}\right)}\|y-F(x(s))\|\left\|L(x(s))\left(x\left(\theta\left(T_{2}\right)\right)-\hat{x}\right)\right\| d s .
\end{aligned}
$$

Applying Assumption (2.2) (c), and the definition of the function $\theta(T)$, it is easy to obtain

$$
\begin{aligned}
\left\|L(x(s))\left(x\left(\theta\left(T_{2}\right)\right)-\hat{x}\right)\right\| & =\left\|L(x(s))\left(x\left(\theta\left(T_{2}\right)\right)-x(s)\right)\right\|+\|L(x(s))(x(s)-\hat{x})\| \\
& \leqslant 3(1+\eta)\|y-F(x(s))\| .
\end{aligned}
$$

Therefore, combining the property $\lim _{T \rightarrow \infty}\|F(x(T))-y\|=0$, there exists a constant (still denote as) $\bar{T}$, for $T_{2}>T_{1}>\bar{T}$,

$$
\left|\left\langle\xi\left(\theta\left(T_{1}\right)\right)-\xi\left(\theta\left(T_{2}\right)\right), x\left(\theta\left(T_{2}\right)\right)-\hat{x}\right\rangle\right| \leqslant 3(1+\eta) \int_{\theta\left(T_{1}\right)}^{\theta\left(T_{2}\right)}\|y-F(x(s))\|^{2} d s<\frac{\varepsilon}{3},
$$

and consequently,

$$
D_{\xi\left(\theta\left(T_{1}\right)\right)} \Theta\left(x\left(\theta\left(T_{2}\right)\right), x\left(\theta\left(T_{1}\right)\right)\right)<\left(a+\frac{\varepsilon}{3}\right)-\left(a-\frac{\varepsilon}{3}\right)+\frac{\varepsilon}{3}=\varepsilon .
$$

The application of the $p$-convexity of $\Theta$ implies $\lim _{T \rightarrow \infty} x(\theta(T))$ exists, we denote it as $\bar{x}$.

Similarly, we consider

$$
\begin{aligned}
& D_{\xi(\theta(T))} \Theta(x(\theta(T)), x(T)) \\
& =D_{\xi(\theta(T))} \Theta(\hat{x}, x(\theta(T)))-D_{\xi(T)} \Theta(\hat{x}, x(T))+\langle\xi(T)-\xi(\theta(T)), x(\theta(T))-\hat{x}\rangle
\end{aligned}
$$

Since $\theta(T) \leqslant T$ and $\|F(x(\theta(T)))-y\| \leqslant\|F(x(T))-y\|$, we have

$$
\begin{aligned}
|\langle\xi(T)-\xi(\theta(T)), x(\theta(T))-\hat{x}\rangle| & \leqslant\left|\left\langle\int_{\theta(T)}^{T} L(x(s))^{*}(F(x(s))-y) d s, x(\theta(T))-\hat{x}\right\rangle\right| \\
& \leqslant \int_{\theta(T)}^{T}\|F(x(s))-y\|\|L(x(s))(x(\theta(T))-\hat{x})\| d s \\
& \leqslant 3(1+\eta) \int_{\theta(T)}^{T}\|y-F(x(s))\|^{2} d s .
\end{aligned}
$$


The same technique provides

$$
\lim _{T \rightarrow \infty}|\langle\xi(\theta(T))-\xi(T), x(\theta(T))-\hat{x}\rangle|=0
$$

and consequently,

$$
\lim _{T \rightarrow \infty} D_{\xi(\theta(T))} \Theta(x(\theta(T)), x(T)) \rightarrow 0
$$

and $\lim _{T \rightarrow \infty}\|x(T)-x(\theta(T))\|=0$. The application of triangle inequality gives

$$
\|x(T)-\bar{x}\| \leqslant\|x(\theta(T))-\bar{x}\|+\|x(T)-x(\theta(T))\|,
$$

therefore,

$$
\lim _{T \rightarrow \infty} x(T)=\bar{x} .
$$

By the weakly compactness of $F$ and continuity of $x(T)$, we can conclude $\bar{x}$ is a solution to (1.1) and $\bar{x} \in B_{2 \rho}\left(x_{0}\right) \bigcap D(\Theta)$.

Finally, we perform the analysis from the Hölder continuity of the inverse mapping instead of the source and non-linearity conditions and obtain the convergence rates.

Assumption 3.6. Assume the Hölder type conditional stability, i.e., denote the $\nu \in\left[\frac{p}{2}, p\right]$, there exists a constant $R_{F}$ such that

$$
D_{\bar{\xi}} \Theta(x, \bar{x}) \leqslant R_{F}\|F(x)-F(\bar{x})\|^{\nu}, \quad \forall x, \bar{x} \in B_{2 \rho}\left(x_{0}\right), \bar{x} \in \mathcal{D}(\partial \Theta), \bar{\xi} \in \partial \Theta(\bar{x}) .
$$

Theorem 3.7. Let Assumption 2.2, in which the tangential cone condition is replaced by Lipschitz continuity of $F^{\prime}$

$$
\left\|F^{\prime}(x)-F^{\prime}(\bar{x})\right\| \leqslant L^{\prime}\|x-\bar{x}\|, \quad x, \bar{x} \in B_{2 \rho}\left(x_{0}\right) .
$$

and Assumption 3.6 hold true. Assume $\left\|F\left(x_{0}\right)-y^{\delta}\right\|>\tau \delta$, let $\left(\xi^{\delta}(T), x^{\delta}(T)\right)$ be the solution of (3.1) with $L(x)=F^{\prime}(x)$, where $T=T^{*}$ is the solution of (3.8). Then, there is a solution $\bar{x}$ of (1.1) in $B_{2 \rho}\left(x_{0}\right)$, there holds

$$
D_{\xi^{\delta}\left(T^{*}\right)}^{\delta} \Theta\left(\bar{x}, x^{\delta}\left(T^{*}\right)\right) \rightarrow 0, \quad \text { as } \delta \rightarrow 0
$$

and the convergence rate is given by

$$
D_{\xi^{\delta}\left(T^{*}\right)}^{\delta} \Theta\left(\bar{x}, x^{\delta}\left(T^{*}\right)\right) \leqslant R_{F}(\tau+1)^{\nu} \delta^{\nu} .
$$

Proof. Assume $x^{\delta}(T) \in B_{2 \rho}\left(x_{0}\right)$ for $T<T^{*}$, we have

$$
\begin{aligned}
\left\|y-F\left(x^{\delta}(T)\right)-F^{\prime}\left(x^{\delta}(T)\right)\left(\hat{x}-x^{\delta}(T)\right)\right\| & \leqslant \frac{L}{2}\left\|x^{\delta}(T)-\hat{x}\right\|^{2} \\
& \leqslant \frac{L}{2}\left\|x^{\delta}(T)-\hat{x}\right\|^{2-\frac{p}{\nu}}\left\|x^{\delta}(T)-\hat{x}\right\|^{\frac{p}{\nu}} \\
& \leqslant \frac{L}{2}(4 \rho)^{2-\frac{p}{\nu}}\left(\frac{1}{c_{0}} D_{\xi^{\delta}\left(T_{n}\right)} \Theta\left(\hat{x}, x^{\delta}\left(T_{n}\right)\right)\right)^{\frac{1}{\nu}} \\
& \leqslant \frac{L}{2}(4 \rho)^{2-\frac{p}{\nu}}\left(\frac{R_{F}}{c_{0}}\right)^{\frac{1}{\nu}}\left\|y-F\left(x^{\delta}\left(T_{n}\right)\right)\right\| .
\end{aligned}
$$

Denote

$$
\rho=\frac{1}{4}\left(\frac{2 \eta}{L}\right)^{\frac{\nu}{2 \nu-p}}\left(\frac{c_{0}}{R_{F}}\right)^{\frac{1}{2 \nu-p}}
$$

then Assumption 3.6 with $p / 2 \leqslant \nu \leqslant p$ and (3.17) imply the general tangential cone condition. The conclusion is directly obtained by utilizing the conditional stability 
in Assumption 3.6 .

$$
\begin{aligned}
D_{\xi^{\delta}\left(T^{*}\right)}^{\delta} \Theta\left(\bar{x}, x^{\delta}\left(T^{*}\right)\right) & \leqslant R_{F}\left\|y-F\left(x^{\delta}\left(T^{*}\right)\right)\right\|^{\nu} \\
& \leqslant R_{F}\left(\delta+\left\|y^{\delta}-F\left(x^{\delta}\left(T^{*}\right)\right)\right\|\right)^{\nu} \\
& \leqslant R_{F}(\tau+1)^{\nu} \delta^{\nu} .
\end{aligned}
$$

Remark 3.8. Note that one obtains for finite $T<\infty$, the existence of a unique solution $\xi(t) \in C(0, T ; \mathcal{X})$ of (3.1) if the operator $G(\xi)=F^{\prime}\left(\nabla \Theta^{*}(\xi)\right)^{*}\left[y^{\delta}-\right.$ $F\left(\nabla \Theta^{*}(\xi)\right)$ ] is locally Lipschitz continuous in $\mathcal{X}$ [8]. Actually, for $\left(\xi_{1}, x_{1}\right)$ and $\left(\xi_{2}, x_{2}\right)$ with $x_{i}=\nabla \Theta^{*}\left(\xi_{i}\right), i=1,2$, and $x_{1}, x_{2} \in B_{2 \rho}\left(x_{0}\right)$, we have

$$
\begin{aligned}
G\left(\xi_{1}\right)-G\left(\xi_{2}\right) & =F^{\prime}\left(x_{1}\right)^{*}\left(y^{\delta}-F\left(x_{1}\right)\right)-F^{\prime}\left(x_{2}\right)^{*}\left(y^{\delta}-F\left(x_{2}\right)\right) \\
& =\left(F^{\prime}\left(x_{1}\right)^{*}-F^{\prime}\left(x_{2}\right)^{*}\right)\left(y^{\delta}-F\left(x_{1}\right)\right)+F^{\prime}\left(x_{2}\right)^{*}\left(F\left(x_{2}\right)-F\left(x_{1}\right)\right) d t,
\end{aligned}
$$

Applying the Lipschitz continuity of $F^{\prime}$ (3.17) and the bounded property of $F^{\prime}$, then we have

$$
\begin{aligned}
\left\|G\left(\xi_{1}\right)-G\left(\xi_{2}\right)\right\| & \leqslant\left\|\left(F^{\prime}\left(x_{1}\right)^{*}-F^{\prime}\left(x_{2}\right)^{*}\right)\left(y^{\delta}-F\left(x_{1}\right)\right)\right\|\left\|F^{\prime}\left(x_{2}\right)^{*}\left(F\left(x_{2}\right)-F\left(x_{1}\right)\right)\right\| \\
& \leqslant L^{\prime}\left\|y^{\delta}-F\left(x_{1}\right)\right\|\left\|x_{1}-x_{2}\right\|+C_{0}^{2}\left\|x_{1}-x_{2}\right\|
\end{aligned}
$$

Take the $\Theta$ as strongly convex ( $p=2$ ) then

$$
\left\|x_{1}-x_{2}\right\|=\left\|\nabla \Theta^{*}\left(\xi_{1}\right)-\nabla \Theta^{*}\left(\xi_{2}\right)\right\| \leqslant \frac{\left\|\xi_{1}-\xi_{2}\right\|}{2 c_{0}} . \quad \forall \xi_{1}, \xi_{2} \in \mathcal{X} .
$$

Combining above estimates, we obtain that $G(\xi)$ is locally Lipschitz continuous in $\mathcal{X}$, which implies that the existence of a unique solution $\xi(t) \in C(0, T ; \mathcal{X})$ of (3.1).

\section{Discretization of asymptotical method}

In this section we recall the Runge-Kutta (RK) type iterative methods which are regarded as the discretization of asymptotical method.

RK methods are a class of one-step methods for the numerical solutions of the initial value problems for ODEs,

$$
\frac{d w(t)}{d t}=\Psi(t, w(t)), \quad t>0, \quad w(t)=w_{0},
$$

with given $\Psi:[0, \infty] \times X \rightarrow X$ and $w_{0} \in X$.

A RK method of $s$-stages provides approximations $w_{n}$ to $w\left(t_{n}\right)$ with $t_{n}=\sum_{k=1}^{n} \Delta t_{k}$ such that

$$
\begin{aligned}
& w_{n+1}=w_{n}+\triangle t_{n} \sum_{i=1}^{s} b_{i} \Psi\left(t+c_{i} \triangle t_{n}, k_{i}\right), \\
& k_{i}=w_{n}+\triangle t_{n} \sum_{j=1}^{s} a_{i, j} \Psi\left(t+c_{j} \triangle t_{n}, k_{j}\right) .
\end{aligned}
$$

The given coefficients $A=\left(a_{i j}\right) \in \mathbb{R}^{s \times s}, b=\left(b_{1}, b_{2}, \cdots, b_{s}\right)^{T}$ and $c=\left(c_{1}, c_{2}, \cdots, c_{s}\right)^{T}$ determine the particular method. The method is called explicit if $A$ is strictly lower triangular matrix. Otherwise, the method is called implicit, since linear or nonlinear algebraic equations have to be solved to calculate $k_{i}, i=1, \cdots, s$. Usually, the specific instance of RK methods can be presented by Butcher tableau, see Table 1 or by triple $(A, b, c)$ in 8 . We only consider consistent $s$-stage RK method, i.e., $\sum_{i=1}^{s} b_{i}=1$. However, an inconsistent $s$-stage RK method can be considered in the same manner. 
TABLE 1. The general Butcher tableau of RK method.
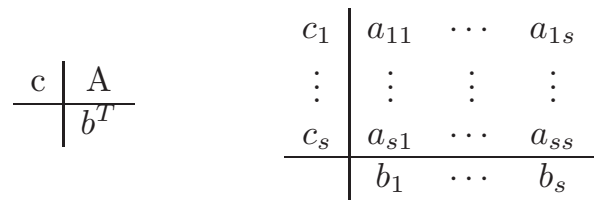

A RK method applied to autonomous ODE (3.1), that is,

$$
\Psi\left(\xi^{\delta}(t)\right)=L\left(x^{\delta}(t)\right)^{*}\left(y^{\delta}-F\left(x^{\delta}(t)\right)\right), \quad x^{\delta}(t)=\nabla \Theta^{*}\left(\xi^{\delta}(t)\right) .
$$

Then, it is consequently

$$
\begin{aligned}
& \xi_{n+1}^{\delta}=\xi_{n}^{\delta}+\triangle t_{n} \sum_{i=1}^{s} b_{i} L\left(\nabla \Theta^{*}\left(k_{i}\right)\right)^{*}\left(y^{\delta}-F\left(\nabla \Theta^{*}\left(k_{i}\right)\right)\right), \\
& k_{i}=\xi_{n}^{\delta}+\triangle t_{n} \sum_{j=1}^{s} a_{i j} L\left(\nabla \Theta^{*}\left(k_{j}\right)\right)^{*}\left(y^{\delta}-F\left(\nabla \Theta^{*}\left(k_{j}\right)\right)\right) .
\end{aligned}
$$

Use the explicit Euler method, i.e., $s=1, c=0, b=1, A=0$, it follow that

$$
\begin{aligned}
& \xi_{n+1}^{\delta}=\xi_{n}^{\delta}+\triangle t_{n} L\left(x_{n}^{\delta}\right)^{*}\left(y^{\delta}-F\left(x_{n}^{\delta}\right)\right), \\
& x_{n+1}^{\delta}=\nabla \Theta\left(\xi_{n+1}^{\delta}\right) .
\end{aligned}
$$

It is a Landweber-type iteration. Use the implicit Euler method with $s=1, c=1$, $b=1$ and $A=1$, we have the implicit Landweber-type iteration

$$
\begin{aligned}
\xi_{n+1}^{\delta} & =\xi_{n}^{\delta}+\triangle t_{n} L\left(x_{n+1}^{\delta}\right)^{*}\left(F\left(x_{n+1}^{\delta}\right)-y^{\delta}\right), \\
x_{n+1}^{\delta} & =\nabla \Theta^{*}\left(\xi_{n+1}^{\delta}\right) .
\end{aligned}
$$

For $s=2$, the explicit 2-stage RK methods provides

$$
\begin{aligned}
& k_{2}=\xi_{n}^{\delta}+\triangle t_{n} a_{21} L\left(x_{n}^{\delta}\right)^{*}\left(y^{\delta}-F\left(x_{n}^{\delta}\right)\right), \\
& z_{n}^{\delta}=\nabla \Theta^{*}\left(k_{2}\right), \\
& \xi_{n+1}^{\delta}=\xi_{n}+\triangle t_{n}\left(b_{1}\left(y^{\delta}-F\left(x_{n}^{\delta}\right)\right)+b_{2} L\left(z_{n}^{\delta}\right)^{*}\left(y^{\delta}-F\left(z_{n}^{\delta}\right)\right)\right), \\
& x_{n+1}^{\delta}=\nabla \Theta^{*}\left(\xi_{n+1}^{\delta}\right),
\end{aligned}
$$

in which the coefficients should satisfy

$$
b_{1}+b_{2}=1, \quad a_{21} b_{2}=\frac{1}{2} .
$$

\section{Acknowledgements}

The work of M Zhong is supported by the National Natural Science Foundation of China (No. 11871149) and supported by Zhishan Youth Scholar Program of SEU. The work of W Wang is supported by the National Natural Science Foundation of China (No. 12071184).

\section{REFERENCES}

[1] R. Bogt, G. Dong, P. Elbau and O. Scherzer, Convergence rates of first-and higher-order dynamics for solving linear ill-posed problems. Foundations of Computational Mathematics. 2021 https://doi.org/10.1007/s10208-021-09536-6.

[2] R. Bot and T. Hein, Iterative regularization with a geeral penalty term: theory and applications to $L^{1}$ and TV regularization, Inverse Problems 28 (2012) 104010.

[3] C. Bckmann, P. Pornsawad, Iterative Runge-Kutta-type methods for nonlinear ill-posed problems. Inverse Problems 24(2) (2008) 025002.

[4] A. Beck and M. Teboulle, A fast iterative shrinkage-thresholding algorithm for linear inverse problems, SIAM J. Imag. Sci. 2 (2009) 183-202. 
[5] A. Beck and M. Teboulle, Fast gradient-based algorithms for constrained total variation image denoising and deblurring problems, IEEE Trans. Image Process. 18 (2009) 2419-2434.

[6] J. Cheng, B. Hofmann and S. Lu, The index function and Tikhonov regularization for ill-posed problems, J. Comput. Appl. Math. 265 (2014) 110-119.

[7] J. Cheng and Y. Yamamoto, One new strategy for a priori choice of regularizing parameters in Tikhonov's regularization, Inverse Problems 16 (2008) L31-L38.

[8] P. Deuflhard and F. Bornemann, Scientific Computing with Ordinary Differential Equations, New York: Springer, 2002.

[9] H. Engl, M. Hanke, A. Neubauer, Regularization of Inverse Problems, New York (NY): Springer, 1996

[10] R. Gu, B. Han and Y. Chen, Fast subspace optimization method for nonlinear inverse problems in Banach spaces with uniformly convex penalty terms, Inverse Problems 35 (2019) 125011.

[11] M. V. de Hoop, L. Qiu, O. Scherzer, Local analysis of inverse problems: Hölder stability and iterative reconstruction, Inverse Problems 28 (2012) 045001.

[12] M. Hanke, A regularizing Levenberg Marquardt scheme with applications to inverse groundwater filtration problems, Inverse Problems 13 (1997) 79-95.

[13] M. Hanke, A. Neubauer and O. Scherzer, A convergence analysis of the Landweber iteration for nonlinear ill-posed problems, Numer. Math. 72 (1995) 21-37.

[14] Q. Jin, Inexact Newton-Landweber iteration in Banach spaces with nonsmooth convex penalty terms, SIAM J. Numer. Anal. 53 (5) (2015) 2389-2413.

[15] Q. Jin, Landweber-Kaczmarz method in Banach spaces with inexact inner solvers, Inverse Problems 32 (2016) 104005.

[16] Q. Jin, and W. Wang, Landweber iteration of Kaczmarz type with general non-smooth convex penalty functionals, Inverse Problems 29 (2013) 085011.

[17] L. Li, B. Han and W. Wang, R-K type Landweber method for nonlinear ill-posed problems, J. Comput. Appl. Math. 206 (2007) 341-357.

[18] S. Lu, P. Niu and F. Werner, On the asymptotical regularization for linear inverse problems in presence of white noise, SIAM/ASA Journal on Uncertainty Quantification 9(1) (2021) $1-28$.

[19] P. Maaß and R. Strehlow, An iterative regularization method for nonlinear problems based on Bregman projections, Inverse Problems 32 (2016) 115013.

[20] A. Rieder, On the regularization of nonlinear ill-posed problems via inexact Newton iterations, Inverse Problems, 15 (1999) 309-327.

[21] L. Rudin, S. Osher and C. Fatemi, Nonlinear total variation based noise removal algorithm, Phys. D 60 (1992) 259-268.

[22] A. Rieder, Runge-Kutta integrators yield optimal regularization schemes, Inverse Problems 21 (2005) 453-471.

[23] R. Real and Q. Jin, A revisit on Landweber iteration, Inverse Problems 36 (2020) 075011.

[24] U. Tautenhahn, On the asymptotical regularization of nonlinear ill-posed problems, Inverse Problems 10 (1994) 1405-1418.

[25] C. Zălinscu, Convex Analysis in General Vector Spaces World Scientific Publishing Co., Inc., River Edge. 2002.

[26] Y. Zhao, P. Mathé, S. Lu, Convergence analysis of asymptotical regularization and RungeKutta integrators for linear inverse problems under variational source conditions, CSIAM Transactions on Applied Mathematics 1(4) (2020) 693-714.

[27] M. Zhong, L. Qiu, W. Wang, Landweber-type method with uniformly convex constraints under conditional stability assumptions, preprint.

[28] M. Zhong, W. Wang, The two-point gradient methods for nonlinear inverse problems based on Bregman projections, Inverse Problems 36 (2020) 045012.

[29] M. Zhong, W. Wang and Q. Jin, Regularization of inverse problems by two-point gradient methods in Banach spaces, Numer. Math. 143 (2019) 713-747.

School of Mathematics, Southeast University, Nanjing, Jiangsu 210096, P. R. China Email address: min.zhong@seu.edu.cn

Corresponding author. College of Mathematics, Physics and Information Engineering, Jiaxing University, Zhejiang 314001, P. R. China

Email address: weiwang@zjxu.edu.cn 